\title{
A prevalence study of bifid mandibular canals using cone-beam computed tomography
}

Prevalence study of bifid mandibular canals

James R Allison ${ }^{1}$, Andrew Carr ${ }^{2}$

\author{
${ }^{1}$ BDS(Hons), MFDS RCPS Glasg \\ General Professional Trainee \\ Newcastle Dental Hospital \\ Richardson Road \\ Newcastle upon Tyne \\ Tyne and Wear \\ NE2 4AZ \\ England
}

${ }^{2}$ BDS, MFDS RCSEd, PGCert

Specialty Registrar and Associate

Clinical Lecturer in Dental and

Maxillofacial Radiology

Newcastle Dental Hospital

Richardson Road Newcastle upon Tyne

Tyne and Wear

NE2 4AZ

England

This is the peer reviewed version of the following article: [Allison, J. and Carr, A. (2017), A prevalence study of bifid mandibular canals using cone beam computed tomography. Oral Surg, 10: e55-e61.] which has been published in final form at [doi:10.1111/ors.12267]. This article may be used for non-commercial purposes in accordance with Wiley Terms and Conditions for Use of Self-Archived Versions

\author{
Submitted - 25 $5^{\text {th }}$ October 2016 \\ Revision Submitted $-27^{\text {th }}$ November 2016
}

\section{Declarations}

The authors declare that there are no conflicts of interest. This research did not receive any specific funding from funding agencies in the public, commercial or not-for-profit sectors. On the advice of the local research ethics committee, it was felt ethical approval was not necessary due to the nature of the research. Reporting complies with the STROBE statement for cross-sectional studies. 


\section{Abstract}

Aim

This study aims to assess the prevalence of bifid mandibular canals in a UK population using cone beam computed tomography.

\section{Materials and Methods}

This is a prevalence study of 140 patients ( 280 sides) referred to a dental hospital in the UK for extraction of a third molar. Canals were classified from cone beam computed tomography images using a novel classification.

\section{Results}

At least one bifid canal was seen in $30.00 \%(n=42 / 140)$ of patients $(95 \% \mathrm{Cl}: 26.13-33.87 \%)$ and in $18.93 \%(n=53 / 280)$ of sides $(95 \% \mathrm{Cl}: 16.59-21.27 \%)$. The retromolar canal was the most common variant observed.

\section{Conclusions}

The bifid mandibular canal appears to be a common anatomical variant, of which clinicians should be aware. These variants are relevant to surgery in the retromolar region, dental extraction and implant placement, where damage has the potential to cause bleeding and sensory disturbance.

\section{Clinical Relevance}

\section{Scientific Rationale for Study}

A case of prolonged post-extraction bleeding from a third-molar socket in our unit alerted us to investigate bifid mandibular canals. These variants are thought by many to be quite rare, and it was our aim to test this assumption, particularly as the prevalence had not been investigated in a UK population before.

\section{Principal Findings}

We found that bifid canals are actually relatively common, with $30 \%$ of patients having at least one.

\section{Practical Implications}

As we discuss, the presence of a bifid canal has the potential to cause complications if not properly identified. The reader should be able to appreciate their significance.

Keywords: cone beam; computed tomography; bifid mandibular canal; retromolar canal; prevalence; inferior alveolar nerve; third molar 


\section{Introduction}

A female patient in her 20s underwent an uncomplicated surgical extraction of a horizontally impacted lower third molar as an outpatient in the authors' unit under local anaesthesia and was discharged without incident. The patient subsequently presented on four separate occasions to the emergency department with significant uncontrolled bleeding from the operative site, requiring admission on two of these occasions. Blood investigations showed no abnormality and she was treated by local haemostatic measures and with oral tranexamic acid. Cone beam computed tomography (CBCT) imaging was performed as a further investigation and showed the presence of an "aberrant" canal opening into the socket of the recently extracted third molar which had neither been appreciated preoperatively, nor at the time of surgery, and had likely been the cause of the subsequent haemorrhage. It was this case that alerted the authors to anatomical variations of the mandibular canal as an area of further study.

"Bifid mandibular canal" is a term encompassing several anatomical variants of the mandibular canal and could be defined as a canal which divides into two discernible parts at some position along its course between the mandibular and mental foramina. Smaller canals branching from the main body of the mandibular canal have been shown in histological studies to contain neural, arterial and venous structures ${ }^{1,2}$.

There have been several systems proposed for the classification of bifid mandibular canals, the earliest of which were based on dental panoramic radiography (DPR) ${ }^{3,4}$. The reported prevalence of bifid canals identified by DPR is between $0.35 \%$ and $1.98 \%$ of patients ${ }^{3-7}$. The prevalence reported in CBCT studies however is much greater, at $10.2-66.5 \%$ of patients $(10.2-47.4 \% \text { of sides })^{8-19}$. This would imply that DPR has a low sensitivity for identifying such anatomy. Naitoh et al. ${ }^{12}$ proposed a system of classification for bifid mandibular canals based on CBCT which has been used or adapted in several studies ${ }^{9-13,15}$.

The present study aims to investigate the prevalence of bifid mandibular canals. The authors propose a novel classification based upon a modification of that proposed by Naitoh et al. ${ }^{12}$. This study is, to the authors' knowledge, the first to investigate the prevalence of bifid canals in a UK population.

\section{Materials and Methods}

The present study is a prevalence study of bifid mandibular canals in a population of patients referred to the Oral Surgery department of a dental hospital in the north of England for extraction of a third molar. Imaging was undertaken in the Dental and Maxillofacial Radiology department of the same hospital.

\section{Participants}

Data collection began in July 2015 , by retrospectively searching the hospital picture archiving and communication system to identify all CBCT investigations undertaken between March and June 2015. Cases were included only if the images were taken prior to planned surgical extraction of a third molar, and had been justified in accordance with IR(ME)R 2000. Cases were excluded if the 
images did not clearly show the full length of the mandibular canal from mandibular to mental foramen. To increase the number of cases included we also prospectively identified cases as they were referred to the Radiology department between August and September 2015, using the same inclusion criteria.

\section{Imaging}

Cone beam CT examinations were performed using a Newtom VGi (QR S.R.L., Verona, Italy) machine. Each dataset was reviewed on a Philips Brilliance 19S monitor linked to a HP Z600 modality workstation. The modality software utilised in this study was NNT version 5.3.3 (QR S.R.L., Verona, Italy) to produce primary data reconstruction and multiplanar reformation images of the original, uncompressed volumetric data sets.

\section{Canal Classification}

Both right and left mandibular canals were viewed, firstly in the transaxial plane, and then in the coronal plane to assess the location of and classify the canals. The canals were assessed using a novel classification based on a modification to that proposed by Naitoh et al. ${ }^{12}$ which is summarised in Table 1. Classification was performed jointly by one senior Specialty Registrar in Dental and Maxillofacial Radiology with 5 years of experience in $C B C T$ imaging $(A C)$ and one Dental Core Trainee (JRA). Images were viewed in optimal conditions and graded by both examiners together at the same time. Any disagreement between examiners was resolved by discussion until consensus was reached. Examiners were not blinded to the identity of patients.

\section{Statistical Methods}

Descriptive and comparative statistics were calculated using OpenStat (WG Miller, 2014) and Microsoft Excel (Microsoft Corporation, Washington, USA 2010). An a priori sample size calculation suggested a sample size of 139 participants based on an estimated prevalence of $10 \%$, confidence level of $95 \%$ and precision of $5 \%$. This was rounded to 140 participants. $95 \%$ confidence intervals were calculated for reported prevalences. A two-proportion z-test was used to compare the proportion of bifid canals between gender. Because the data for left and right sides was paired, and therefore non-independent, a McNemar test was used to compare the proportion of bifid canals between sides. Significance was set at $p<0.05$ for all tests.

\section{$\underline{\text { Results }}$}

A total of 140 patients were included in the study. All data sets analysed included the left and right mandibular canals meaning 280 sides were analysed. $74.3 \%$ of patients were female. The mean age was 34.9 years (SD:12.2) and the range was $16-73$ years.

At least one bifid mandibular canal of any type was present in in $30.00 \%(n=42 / 140)$ of patients (95\% $\mathrm{Cl}: 26.13-33.87 \%) ; 11$ patients had bilateral bifid canals. A bifid canal of any type was present in $18.93 \%(n=53 / 280)$ of sides (95\% Cl:16.59-21.27\%). 
There was no statistically significant difference in the proportion of bifid canals between genders $(z=-1.35$, two-tailed $p=0.177)$, however the proportion was significantly greater in right hemimandibles (two-tailed $p=0.011$ ).

The retromolar canal was the most common variant observed. Full results and canal types are given in Table 2.

\section{Discussion}

The presence of a bifid mandibular canal may be clinically relevant in several scenarios: any surgery in the retromolar region of the mandible, such as surgical removal of a third molar or sagittal split osteotomy, has the potential to damage structures emerging from a retromolar canal. Similarly, prostheses compressing nerve fibres emerging from such a canal could result in pain. The contents of the retromolar canal have been found to include neural, venous and arterial structures ${ }^{1,2}$ and there is therefore the potential for bleeding and sensory disturbance if damaged. It is unclear what distribution the retromolar nerve fibres supply; perhaps they are branches of the inferior alveolar nerve (IAN) leaving the mandible, as work by Schejtman, Devoto, and Arias ${ }^{20}$ would suggest. Conversely they may contain fibres from other branches of the trigeminal nerve which enter the mandible and give sensory supply to the teeth and other structures; this could perhaps cause difficulty in achieving dental anaesthesia with an IAN block alone. The dental-type canal may be a potential cause of bleeding following dental extraction if the vessels contained within are of a large calibre and become damaged during the procedure; this was deemed to have been so in the case discussed in the introduction. A forward or buccolingual type canal may be damaged by implant placement if not appreciated preoperatively ${ }^{21}$.

The Naitoh classification was chosen for use in the present study as it is also based on CBCT imaging. Initially, Carter and Keen ${ }^{22}$ described a classification of variations of the mandibular canal based on the dissection of cadaveric mandibles, which included three canal arrangements: single canal, inferiorly placed canal and duplicated canal. Building on this work, Nortje et al. ${ }^{3}$ later classified bifid canals using DPR into three types: two canals originating from one foramen, a second canal extending to the molar teeth, or two canals arising from separate mandibular foramina. Similarly, Langalis et al. ${ }^{4}$ used DPR to classify canals according to their location and the course of the branches.

As previously mentioned, the low sensitivity of DPR for imaging bifid mandibular canals makes the modality inferior to CBCT in this regard. The added benefit of CBCT is that the patient's data set can be viewed in 3 orthogonal planes, and the inherent weakness of summation when using DPR is removed. DPR is a tomographic technique, and therefore relies on imaging a "slice" through the patient via the focal trough. Errors in operator technique may lead to problems with $\mathrm{x}$-ray beam angulation, $x$-ray source-to-object distances and malpositioning with respect to the focal trough. In light of this, resultant anatomy may be misrepresented or distorted, which may then affect the interpretation of the image. It is worth noting that radiation doses for $\mathrm{CBCT}$ investigations are greater than for DPR, and the modality is not be appropriate in every case; a recent systematic review of $C B C T$ guidelines was published by Horner et al. ${ }^{23}$ and serves as a useful source when considering radiographic selection criteria. 
Naitoh et al. ${ }^{12}$ used CBCT to classify canals into four types: retromolar, dental, forward and buccolingual, however they did not include a category for bifid mandibular canals arising from an accessory mandibular foramen (AMF) ${ }^{3,4}$, which is a recognised anatomical variant seen in around $1 \%$ patients $^{24}$. A canal arising from an AMF would appear separate to the main mandibular canal, entering the mandible via a separate foramen on the medial surface of the ramus. The canal may or may not re-join the main mandibular canal. The authors felt that the addition of an AMF-type to the Naitoh classification would make the system more complete, given the prevalence of this variant.

In the authors' unit, patients only receive a CBCT investigation prior to third molar surgery where DPR indicates an intimate relationship of the mandibular canal to the roots of the teeth, where the canal is not discernible, or where there is deemed to be any other clinical benefit to CBCT imaging. Additionally, only patients who were referred to secondary care for third molar extraction were included. Inclusion of patients who were imaged for any indication may make any findings more externally valid and it would be interesting to see if the findings of such a study differ from the present one. For these reasons, it is possible that the sample prevalence in this study is greater than the true population prevalence; however we feel our findings are valid in the population studied.

Although the sample size was fairly small, the calculated precision was $3.87 \%$ at the patient level and $2.34 \%$ for sides based on a $95 \%$ confidence level; this means one can be fairly certain the sample prevalence approximates the true prevalence in this population.

No other prevalence studies of bifid mandibular canals utilising CBCT have been reported in a UK population, although the findings are in agreement with similar studies in other populations. Studies from Asian populations ${ }^{8,9}, 11,12,14-16$ report prevalences of between $10.2-65 \%$ of patients $(10.2-$ $47.36 \%$ of sides), from European populations ${ }^{13,17} 19-36.8 \%$ of patients ( $22.8 \%$ of sides), and from other populations ${ }^{10,18} 19.68-66.5 \%$ of patients ( $46.5 \%$ of sides). Studies looking specifically at retromolar canals ${ }^{9-11}, 13-15,17,25-29$ report prevalences of between $1.66-75.4 \%$ compared to the $10.00 \%$ found in the present study. Of the studies using the Naitoh classification ${ }^{9-13,15}$ the retromolar or forward types were the most common variants observed. The large variation in reported prevalences could be explained to some degree by the disparate populations in which the studies were carried out, however the lack of standardisation between assessment criteria, experience of the assessor and the differences in radiographic equipment used likely explain some of the heterogenicity.

In conclusion, although often missed on DPR, the bifid mandibular canal appears to be a common anatomical variant in this population and one which clinicians should be familiar with. All canal types may be clinically relevant in different scenarios, and in the present study the retromolar type was most common. Although there is large variation in other studies, the present study appears to be in broad agreement with these.

\section{Acknowledgements}

The authors would like to acknowledge Dr lain Macleod for his assistance, and Mr James Adams for his permission to include the introductory case-study and his encouragement. 


\section{$\underline{\text { References }}$}

1. Bilecenoglu B, Tuncer N. Clinical and anatomical study of retromolar foramen and canal. J Oral Maxillofac Surg. 2006;64:1493-7.

2. Fukami K, Shiozaki K, Mishima A, Kuribayashi A, Hamada Y, Kobayashi K. Bifid mandibular canal: confirmation of limited cone beam $\mathrm{CT}$ findings by gross anatomical and histological investigations. Dentomaxillofac Radiol. 2012;41:460-5.

3. Nortje CJ, Farman AG, Grotepass FW. Variations in the normal anatomy of the inferior dental (mandibular) canal: a retrospective study of panoramic radiographs from 3612 routine dental patients. Br J Oral Surg. 1977;15:55-63.

4. Langlais RP, Broadus R, Glass BJ. Bifid mandibular canals in panoramic radiographs. J Am Dent Assoc. 1985;110:923-6.

5. Sanchis JM, Penarrocha M, Soler F. Bifid mandibular canal. J Oral Maxillofac Surg. 2003;61:422-4.

6. Kuczynski A, Kucharski W, Franco A, Westphalen FH, de Lima AA, Fernandes A. Prevalence of bifid mandibular canals in panoramic radiographs: a maxillofacial surgical scope. Surg Radiol Anat.

2014;36:847-50.

7. Kalantar Motamedi MH, Navi F, Sarabi N. Bifid mandibular canals: prevalence and implications. J Oral Maxillofac Surg. 2015;73:387-90.

8. Shen EC, Fu E, Fu MM, Peng M. Configuration and corticalization of the mandibular bifid canal in a Taiwanese adult population: a computed tomography study. Int J Oral Maxillofac Implants. 2014;29:893-7.

9. Rashsuren O, Choi JW, Han WJ, Kim EK. Assessment of bifid and trifid mandibular canals using cone-beam computed tomography. Imaging Sci Dent. 2014;44:229-36.

10. Orhan K, Aksoy S, Bilecenoglu B, Sakul BU, Paksoy CS. Evaluation of bifid mandibular canals with cone-beam computed tomography in a Turkish adult population: a retrospective study. Surg Radiol Anat. 2011;33:501-7.

11. Naitoh M, Nakahara K, Suenaga Y, Gotoh K, Kondo S, Ariji E. Comparison between cone-beam and multislice computed tomography depicting mandibular neurovascular canal structures. Oral Surg Oral Med Oral Pathol Oral Radiol Endod. 2010;109:e25-31.

12. Naitoh M, Hiraiwa Y, Aimiya H, Ariji E. Observation of bifid mandibular canal using cone-beam computerized tomography. Int J Oral Maxillofac Implants. 2009;24:155-9. 
13. Muinelo-Lorenzo J, Suarez-Quintanilla JA, Fernandez-Alonso A, Marsillas-Rascado S, SuarezCunqueiro MM. Descriptive study of the bifid mandibular canals and retromolar foramina: cone beam CT vs panoramic radiography. Dentomaxillofac Radiol. 2014;43:20140090.

14. Kuribayashi A, Watanabe H, Imaizumi A, Tantanapornkul W, Katakami K, Kurabayashi T. Bifid mandibular canals: cone beam computed tomography evaluation. Dentomaxillofac Radiol. 2010;39:235-9.

15. Kang JH, Lee KS, Oh MG, Choi HY, Lee SR, Oh SH, et al. The incidence and configuration of the bifid mandibular canal in Koreans by using cone-beam computed tomography. Imaging Sci Dent. 2014;44:53-60.

16. Fu E, Peng M, Chiang CY, Tu HP, Lin YS, Shen EC. Bifid mandibular canals and the factors associated with their presence: a medical computed tomography evaluation in a Taiwanese population. Clin Oral Implants Res. 2014;25:e64-7.

17. de Oliveira-Santos C, Souza PH, de Azambuja Berti-Couto S, Stinkens L, Moyaert K, Rubira-Bullen $\mathrm{IR}$, et al. Assessment of variations of the mandibular canal through cone beam computed tomography. Clin Oral Investig. 2012;16:387-93.

18. Neves FS, Nascimento MC, Oliveira ML, Almeida SM, Boscolo FN. Comparative analysis of mandibular anatomical variations between panoramic radiography and cone beam computed tomography. Oral Maxillofac Surg. 2014;18:419-24.

19. Yi G, Qiaohong Z, Xiaoqian H. Analysis of bifid mandibular canal via cone beam computed tomography. West China journal of stomatology. 2015;33:158-60.

20. Schejtman R, Devoto FC, Arias NH. The origin and distribution of the elements of the human mandibular retromolar canal. Arch Oral Biol. 1967;12:1261-8.

21. Aljunid S, AlSiweedi S, Nambiar P, Chai WL, Ngeow WC. The Management of Persistent Pain From a Branch of the Trifid Mandibular Canal due to Implant Impingement. J Oral Implantol. 2016;42:34952.

22. Carter RB, Keen EN. The intramandibular course of the inferior alveolar nerve. J Anat. 1971;108:433-40.

23. Horner K, O'Malley L, Taylor K, Glenny AM. Guidelines for clinical use of CBCT: a review. Dentomaxillofac Radiol. 2015;44:20140225.

24. Choi YY, Han SS. Double mandibular foramen leading to the accessory canal on the mandibular ramus. Surg Radiol Anat. 2014;36:851-5. 
25. von Arx T, Hanni A, Sendi P, Buser D, Bornstein MM. Radiographic study of the mandibular retromolar canal: an anatomic structure with clinical importance. J Endod. 2011;37:1630-5.

26. Sisman Y, Ercan-Sekerci A, Payveren-Arikan M, Sahman H. Diagnostic accuracy of cone-beam CT compared with panoramic images in predicting retromolar canal during extraction of impacted mandibular third molars. Med Oral Patol Oral Cir Bucal. 2015;20:e74-81.

27. Patil S, Matsuda Y, Nakajima K, Araki K, Okano T. Retromolar canals as observed on cone-beam computed tomography: their incidence, course, and characteristics. Oral Surg Oral Med Oral Pathol Oral Radiol. 2013;115:692-9.

28. Lizio G, Pelliccioni GA, Ghigi G, Fanelli A, Marchetti C. Radiographic assessment of the mandibular retromolar canal using cone-beam computed tomography. Acta Odontol Scand. 2013;71:650-5.

29. Han SS, Hwang YS. Cone beam CT findings of retromolar canals in a Korean population. Surg Radiol Anat. 2014;36:871-6. 